\title{
Design and Implementation of a Nonlinear PI Predictive Controller for a Grid-Tied Photovoltaic Inverter
}

\author{
Rachid Errouissi, Member, IEEE, Ahmed AI-Durra, Senior Member, IEEE, and S. M. Muyeen, Senior \\ Member, IEEE
}

\begin{abstract}
This paper presents the design, implementation, and performance testing of a nonlinear proportionalintegral (PI) predictive controller (NPIPC) for a grid-tied inverter used in photovoltaic (PV) systems. A conventional cascade structure is adopted to design the proposed controller, where the outer-loop is used to regulate the DC-link voltage, and the inner-loop is designed as a current controller for adjusting the active and reactive powers injected into the grid. For each loop, the controller is derived based on combining a continuous-time nonlinear model predictive control (NMPC) and nonlinear disturbance observer (NDO) techniques. It turns out that the composite controller reduces to a nonlinear $\mathrm{PI}$ controller with a predictive term that plays an important role in improving tracking performance. The salient feature of the proposed approach is its ability to approximately preserve the nominal tracking performance during the startup phase. Both simulation and experimental results are provided to demonstrate the effectiveness of the proposed approach in terms of nominal performance recovery, disturbance rejection, and current control.
\end{abstract}

Index Terms-Continuous-time nonlinear model predictive control (NMPC), disturbance rejection, nonlinear disturbance observer (NDO), nonlinear PI predictive controller (NPIPC), photovoltaic system, renewable energy.

\section{INTRODUCTION}

$\mathbf{P}$ OWER converters are the essence of the renewable energy-based power generation, and it is necessary to ensure reliable and efficient operation of the overall energy conversion system. An inverter is a common interfacing medium used in photovoltaic systems. The main task of an inverter is to control the power exchange between the renewable sources and the grid in terms of voltage and current at system frequency that complies with the grid codes. The phase angle between the current and the voltage is mainly decided by the grid connection requirement. Normally, the phase angle is controlled so that there is no reactive power flow in the ac bus. However, the grid-tied inverter may be forced to tolerate an appropriate reactive power flow to support the grid voltage, e.g., under low-voltage ride through operation [1], [2].

Manuscript received November 25, 2015; revised April 11, 2016, and June 18, 2016; accepted September 05, 2016. This work was supported by "The Petroleum Institute Research Center (PIRC)" Research Grant. R. Errouissi, A. Al-Durra and S. M. Muyeen are with the Petroleum Institute, P.O. Box 2533, Abu Dhabi, United Arab Emirates (e-mails: rerrouissi@pi.ac.ae, aaldurra@pi.ac.ae, and smmuyeen@pi.ac.ae).
Under normal operation, the active power injected in the grid is dependent on atmospheric parameters such as solar irradiation and temperature. Variation of these parameters may lead to power fluctuation, which may cause a large DC-link voltage variation if the PV system is not controlled properly. Hence, the power fluctuation can be considered as a disturbance that affects the DC-link voltage regulation and power quality. Normally, a feedback controller is employed to regulate the DC-link voltage, while at the same time to achieve unity power factor operation despite the presence of model uncertainty and external disturbances.

Various approaches have been proposed for grid-tied power converters, to achieve good transient performances and global stability such as feedback linearization and model predictive control (MPC) [2]-[15]. In [2]-[8], a feedback linearization is employed to control active and reactive power injected in the grid via an inverter. The main drawback of this strategy is that the current cannot be limited as it is considered as an internal dynamics of the closed-loop system. In fact, rapid changes in DC-link voltage reference may cause the current to exceed its limit during transient because of a high voltage derivative. For MPC technique proposed in [9]-[15], the idea is to find the voltage vector minimizing a cost function whose form depends on the performance specifications. To this end, the cost function is evaluated, at each sampling time, for all possible voltage vectors, and the one which gives the lowest cost function is considered as the optimal voltage vector. The main disadvantage of this type of MPC is that a precise knowledge of the model is required to guarantee the robustness of the system plus the high computational effort. Compared to the classical PI controller, both feedback linearization and MPC are still relatively difficult to be practically implemented.

The classical PI controller is widely used under cascaded structure for the regulation of the DC-link voltage and the grid current, as it is capable of rejecting abrupt disturbances and ensuring robustness. The cascaded structure consists of two loops [16], [17]: the outer-loop is used to regulate the DC-link voltage by considering the direct component of the grid current as an input control, whereas the innerloop is employed to track the grid current reference. Hence, the current constraints can easily be handled by limiting the current reference provided by the outer-loop. A look at the literature reveals that there exist several techniques to tune the PI controller parameters with the consideration of 
relative stability and performance. For grid-connected power converters, the tuning rules for the PI controller are often inspired from the symmetrical optimum principle due to its wide stability margin [18]. The drawback of that approach is that the parameters of the controller are mainly dependent on the parameter of the system such as the sampling time, the filter inductor, the DC-link capacitor, the grid voltage, and the DC-link voltage [19]. Generally, this type of tuning is well suited for achieving zero steady-state error but often at the cost of degraded transient performances if the parameters of the system are not accurate.

With a view to provide practitioners with an alternative way to design a PI controller for a grid-tied inverter used in PV systems, a predictive approach is proposed in this work combining continuous-time nonlinear model predictive control (NMPC) and nonlinear disturbance observer (NDO). The composite controller consisting of NMPC and NDO is applied to a grid-tied inverter system under cascaded structure as found in many PV applications. In this work, it is assumed that the grid voltage is balanced and does not experience voltage harmonics. However, the parameters design can also be employed in other existing control schemes, consisting of outer and inner loop such as the proportional resonant controller (PR), to deal with abnormal conditions such as distorted grid voltage [20]. This is because a typical PR controller for a grid-tied inverter usually uses the coefficients of the PI controller [21]. For continuous-time NMPC, the prediction model is approximated via Taylor series expansion up to the relative degree of the nonlinear system [22], which results in a closed-form solution for NMPC problem. The NMPC law is designed based on the nominal model and the desired tracking performance. The nonlinear disturbance observer is incorporated in the control loop to compensate for the unknown disturbances not considered in the nominal model [23]-[27]. It turns out that the simplified NDO contains an integral action, which guarantees zero steady-state error as long as the closedloop system, under the composite controller, is stable. Unlike the classical PI controller, a constant term arises naturally in the controller when deriving the disturbance observer. Such an additional term allows preserving the nominal tracking performance, under the composite controller, during the startup phase. Therefore, excellent tracking performance and fast disturbance rejection can be achieved under the composite controller. Similar strategy is adopted in [28], where a constant term is optimally designed and added to the controller to smooth the system's response during startup phase. However, the major difference here lies in the fact that this term arises naturally in the controller when designing the disturbance observer.

In this work, the design methodology is similar to that used in [27], where Taylor series expansion is adopted to derive a closed-form analytical solution to MPC problem, and a disturbance observer is synthesised to improve the prediction accuracy. But the main difference is that the proposed controller is derived from a nonlinear system and the resulting composite controller is more convenient for practical implementation, as it consists of a PI controller and a prediction term that has the role of improving the dynamic performance of the voltage regulation. However, the work presented in [27] is limited to disturbed linear systems, and requires the integration of the system model as a part of the composite controller, which results in relatively complex control structure.

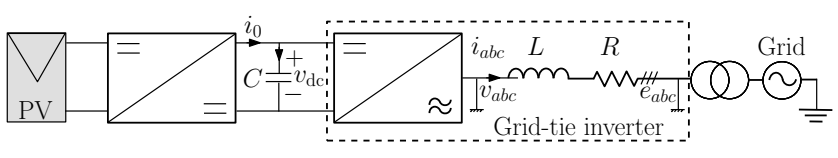

Fig. 1. Schematic diagram of a grid-connected photovoltaic inverter system.

\section{GRID-Tied InVERTER System Modeling}

This work is mainly concerned with the control of the gridtied inverter whose schematic diagram is depicted in Fig. 1. The DC-link voltage $v_{d c}$ is controlled by the dc-ac converter and it is seen as a constant output voltage for the dc-dc converter. The mathematical model of the filter current in the $d-q$ coordinates can be expressed in a bilinear form as follows

$$
\left\{\begin{array}{l}
\frac{d i_{d}}{d t}=-\frac{R}{L} i_{d}+\omega i_{q}+\frac{1}{L} v_{d}-\frac{1}{L} E_{d}-\frac{b_{d}}{L} \\
\frac{d i_{q}}{d t}=-\frac{R}{L} i_{q}-\omega i_{d}+\frac{1}{L} v_{q}-\frac{b_{q}}{L} \\
\frac{d v_{d c}}{d t}=-\frac{3 e_{d}}{2 C v_{d c}} i_{d}-\frac{b_{v}}{C}
\end{array}\right.
$$

where $E_{d}$ is the $d$-axis component of the grid voltage, $i_{d}$ and $i_{q}$ represent respectively the $d$-axis and $q$-axis components of the grid current, and $v_{d}$ and $v_{q}$ are the $d$-axis and $q$-axis components of the voltage at the output of the inverter. The parameter $\omega$ denotes the angular frequency of the grid voltage. Here, it is assumed that $d$-axis component of the grid voltage is forced to be aligned with the fundamental of the grid voltage by regulating the $q$-axis component of the grid voltage to zero. This can be accomplished by using a phase-locked loop (PLL) algorithm [29]. $b_{d}, b_{q}$ and $b_{v}$ are the lumped perturbations caused by model uncertainty and external disturbance such as the current $i_{0}$ provided by the dc-dc converter. $R$ and $L$ are the filter resistance and the filter inductance, respectively, while $C$ is the DC-link capacitance. DC-link voltage $v_{d c}$ and the $q$-axis component $i_{q}$ of the grid current are the variables to be controlled whereas the control inputs are represented by $v_{d}$ and $v_{q}$.

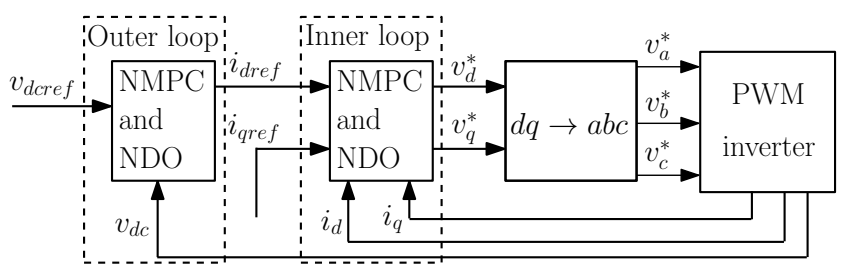

Fig. 2. Control scheme of the cascaded structure.

\section{Control Scheme Using Cascaded Structure}

The structure of the proposed control system is depicted in Fig. 2, from which it can be observed that the control objectives can be met by considering two cascaded control loops. The outer-loop is to track the DC-link voltage reference by considering the $d$-axis component of the grid current as the control input, whereas the inner-loop is used to regulate 
the $d$ and $q$ components of the grid current by generating the $d q$-axis components $v_{d}^{*}$ and $v_{q}^{*}$ of the voltage commands. For each control loop, NMPC approach is combined with a NDO to improve the tracking performance and to eliminate the steady-state error.

\section{NMPC IN THE INNER-LOOP}

\section{A. Design of the Controller}

In the inner current control loop, the continuous-time NMPC is applied to the current equations to provide the $d$ and $q$ components $\left(v_{d}^{*}, v_{q}^{*}\right)$ of the command voltages that minimize the quadratic cost function consisting of the difference between the $d$ and $q$ components of the grid current and their references $i_{\text {dref }}$ and $i_{\text {qref }}$. That is, the quadratic cost function can be expressed as follows

$$
\Im=\frac{1}{2} \int_{0}^{T_{r}} e(t+\tau)^{T} e(t+\tau) d \tau
$$

where $e(\eta)=y_{r}(\eta)-y(\eta)$, and

$$
\left\{\begin{array}{l}
y(t+\tau)=\left[\begin{array}{ll}
i_{d}(t+\tau) & i_{q}(t+\tau)
\end{array}\right]^{T} \\
y_{r}(t+\tau)=\left[\begin{array}{ll}
i_{\text {dref }}(t+\tau) & i_{\text {qref }}(t+\tau)
\end{array}\right]^{T}
\end{array}\right.
$$

and $T_{r}$ represents the predictive time. The input control can be obtained from the necessary condition of optimality

$$
\frac{d \Im}{d u}=0
$$

The first step to design a controller based on the NMPC methodology is to rewrite the current mathematical equations in the nonlinear affine form as

$$
\left\{\begin{array}{l}
\dot{x}=f(x)+g_{1}(x) u(t)+g_{2}(x) b(t) \\
y(t)=h(x)
\end{array}\right.
$$

It follows from (1) that

$$
x=\left[\begin{array}{ll}
i_{d} & i_{q}
\end{array}\right]^{T} ; u=\left[\begin{array}{ll}
v_{d} & v_{q}
\end{array}\right]^{T} ; y=\left[\begin{array}{ll}
i_{d} & i_{q}
\end{array}\right]^{T}
$$

The disturbance vector is given by

$$
b=\left[\begin{array}{ll}
b_{d} & b_{q}
\end{array}\right]^{T}
$$

The input and the disturbance gain matrices $g_{1}$ and $g_{2}$ are given by

$$
g_{1}=\left[\begin{array}{cc}
\frac{1}{L} & 0 \\
0 & \frac{1}{L}
\end{array}\right] ; \quad g_{2}=\left[\begin{array}{cc}
-\frac{1}{L} & 0 \\
0 & -\frac{1}{L}
\end{array}\right]
$$

The vector field $f(x)$ and the output $h(x)$ are defined as

$$
f(x)=\left[\begin{array}{c}
-\frac{R}{L} i_{d}+\omega i_{q}-\frac{E_{d}}{L} \\
-\frac{R}{L} i_{q}-\omega i_{d}
\end{array}\right] ; \quad h(x)=\left[\begin{array}{c}
i_{d} \\
i_{q}
\end{array}\right]
$$

As stated in [22], the next step to follow NMPC methodology is to determine the relative degree $\rho$ for each of the system output with respect to the input. Clearly $\rho$ is equal to the unity. Therefore, the cost function can be simplified by approximating the predicted output and its reference using the first-order Taylor series expansion as follows

$$
y(t+\tau)=H\left[\begin{array}{c}
y(t) \\
\dot{y}(t)
\end{array}\right] ; \quad y_{r}(t+\tau)=H\left[\begin{array}{c}
y_{r}(t) \\
\dot{y}_{r}(t)
\end{array}\right]
$$

where

$$
H=\left[\begin{array}{llll}
1 & 0 & \tau & 0 \\
0 & 1 & 0 & \tau
\end{array}\right]
$$

By considering the numeric value of the relative degree $\rho$, the use of the Lie derivatives $L_{f} h(x)$ and $L_{g_{1,2}} h(x)$ yields

$$
\dot{y}(t)=L_{f} h(x)+L_{g_{1}} h(x) u+L_{g_{2}} h(x) b
$$

Substituting (10)-(12) into (2), and using (4) gives

$$
u=G^{-1}(x)\left(K e+\dot{y}_{r}-L_{f} h(x)-M(x) b\right)
$$

where $e=y_{r}-y$ is the tracking error, and $K$ is the control gain given by

$$
K=K_{0} I_{2 \times 2}, \quad K_{0}=\frac{3}{2 T_{r}}
$$

where $I_{2 \times 2}$ is $2 \times 2$ identity matrix. The matrices $G$ and $M$ are defined by

$$
G=L_{g_{1}} h(x) ; \quad M=L_{g_{2}} h(x)
$$

Substituting the NMPC law (13) in (12) gives to the closedloop system error equations $\dot{e}=-K e$. Clearly, as the predictive time is positive, the closed-loop system under NMPC law is asymptotically stable. Moreover, for a step response, the nominal tracking performance is governed by the closed-loop transfer function

$$
\frac{i_{d}}{i_{\text {dref }}}=\frac{K_{0}}{s+K_{0}} ; \quad \frac{i_{q}}{i_{q r e f}}=\frac{K_{0}}{s+K_{0}}
$$

As the information about the disturbance is not available from direct measurement, the control law (13) can be practically implemented by using the disturbance estimation $\hat{b}$ instead of the actual one $b$. Such an approach may affect the nominal tracking performance if the disturbance observer is not well designed. Thus, the goal is to construct an observer that eliminates the steady-state error and approximately preserves the nominal tracking performance.

\section{B. Design of the Disturbance Observer}

To simplify the design of the disturbance, it is assumed that $b(t)$ is bounded and satisfies

$$
\dot{b}(t)=0
$$

The unknown disturbance can be estimated as follows [23]:

$$
\dot{\hat{b}}(t)=-l(x) g_{2} \hat{b}(t)+l(x)\left(\dot{x}-f(x)-g_{1} u(t)\right)
$$

where $l(x)$ is the observer gain. It follows from (5), (17) and (18), that the disturbance estimation error $e_{b}=b-\hat{b}$ is governed by

$$
\dot{e}_{b}(t)=-l(x) g_{2} e_{b}(t)
$$

Clearly, the stability of the disturbance observer depends on the choice of the observer gain. Indeed, as the disturbance gain $g_{2}$ is a constant matrix, one can choose $l(x)$ as follows

$$
l(x)=\mu \frac{\partial h(x)}{\partial x}=\mu
$$

where $\mu$ is a $2 \times 2$ matrix with constant coefficients, and it can be chosen as $\mu=\operatorname{diag}\left\{\mu_{d}, \mu_{q}\right\}$. Combining (18)-(20), and 
considering the structure of the disturbance matrix gain $g_{2}$, the disturbance observer can be made globally asymptotically stable by choosing $\mu_{d, q}<0$. On the other hand, note that

$$
\begin{cases}l(x) g_{1}(x)=\mu G ; & l(x) g_{2}(x)=\mu M \\ l(x) f(x)=\mu L_{f} h(x) ; & l(x) \dot{x}=\dot{y}\end{cases}
$$

Considering the disturbance estimation $\hat{b}$ and substituting the control law (13) into (18), yields to the simplified observer

$$
\left\{\begin{array}{l}
\dot{\hat{b}}_{d}(t)=-K_{0} \mu_{d} e_{d}(t)-\mu_{d} \dot{e}_{d}(t) \\
\dot{\hat{b}}_{q}(t)=-K_{0} \mu_{q} \dot{e}_{d}(t)-\mu_{q} \dot{e}_{q}(t)
\end{array}\right.
$$

where $e_{d}(t)=i_{\text {dref }}(t)-i_{d}(t)$ and $e_{q}(t)=i_{\text {qref }}(t)-i_{q}(t)$ are the tracking errors. Following [27], in the absence of disturbances, the nominal tracking performance can be preserved under the composite controller by setting $\hat{b}(0)=0$. Thus, integrating (22) gives

$$
\left\{\begin{array}{l}
\hat{b}_{d}(t)=-K_{0} \mu_{d} \int_{0}^{t} e_{d}(\tau) d \tau-\mu_{d} e_{q}(t)+\mu_{d} e_{q}(0) \\
\hat{b}_{q}(t)=-K_{0} \mu_{q} \int_{0}^{t} e_{q}(\tau) d \tau-\mu_{d} e_{q}(t)+\mu_{q} e_{q}(0)
\end{array}\right.
$$

As pointed out in [26], in the presence of lumped disturbances, the composite controller can recover the nominal tracking performance as $\left|\mu_{d, q}\right| \rightarrow \infty$. But from practical standpoint, a large observer gain will eventually magnify the measurement noises. This explains why it is said that the nominal performance can be approximately preserved under the composite controller. Now, substituting (23) into (13) gives the PI predictive controller as follows

$$
\left\{\begin{array}{l}
v_{d}^{*}(t)=P_{d} e_{d}(t)+I_{d} \int_{0}^{t} e_{d}(\tau) d \tau+N_{d}(x) \\
v_{q}^{*}(t)=P_{q} e_{q}(t)+I_{q} \int_{0}^{t} e_{q}(\tau) d \tau+N_{q}(x)
\end{array}\right.
$$

where

$$
P_{d, q}=\left(K_{0} L-\mu_{d, q}\right) ; \quad I_{d, q}=-K_{0} \mu_{d, q}
$$

and

$$
\left\{\begin{array}{l}
N_{d}(x)=L \frac{d i_{d r e f}}{d t}+R i_{d}-L \omega i_{q}+E_{d}+\mu_{d} e_{d}(0) \\
N_{q}(x)=L \frac{d i_{q r e f}}{d t}+R i_{q}+L \omega i_{d}+\mu_{q} e_{q}(0)
\end{array}\right.
$$

Hence, the composite controller can be viewed as a combination between a PI controller and a predictive term $N_{d, q}$, that can predict the error between the system's output and the trajectory to be tracked. In the case of a step response, i.e., $\dot{i}_{\text {dref,qref }}=0$, the term $N_{d, q}$ can be viewed as a feedforward signal to compensate for the grid voltage, the initial tracking error $e_{d, q}(0)$, and for the cross coupling between $d$ and $q$ currents. For a real-time implementation, the time derivative of the currents will not be considered in the controller to avoid magnification of the measurement noises. Thus, by neglecting the filter resistance and the term $e_{d, q}(0)$, the resulting controller becomes exactly equivalent to the conventional decoupled PI controller used for three phase gridconnected renewable energy resources [17].

\section{NMPC IN THE OUTER-LOOP}

The NMPC is applied to the differential equation that describes the dynamics of the DC-link voltage to achieve DC bus voltage regulation. Following (1), the DC-link voltage equation can be written in the form of (5) as

$$
\begin{cases}f(x)=0 ; & h(x)=v_{d c} \\ g_{1}(x)=-\frac{3 E_{d}}{2 C v_{d c}} ; & g_{2}=-\frac{1}{C}\end{cases}
$$

Such a model has the direct $d$-axis current $i_{d}$ as its control input, the DC-link voltage $v_{d c}$ as its output, and $b_{v}$ as a disturbance. Here, the objective is to find the control input that minimizes the cost function (2), with

$$
y(t+\tau)=v_{d c}(t+\tau) ; \quad y_{r}(t+\tau)=v_{d c r e f}(t+\tau)
$$

where $v_{d c r e f}$ is the DC-link voltage reference. The control law can be derived by following the same steps as for the inner-loop. Therefore, as the relative degree with respect to the input is equal to unity, the optimal $d$-axis component of the grid current is given by

$$
i_{d r e f}=G^{-1}(x)\left(K_{0} e+\dot{y}_{r}-L_{f} h(x)-M(x) \hat{b}_{v}\right)
$$

where

$$
G(x)=-\frac{3 E_{d}}{2 C v_{d c}} ; \quad \mathrm{M}=-\frac{1}{C} ; \quad K_{0}=\frac{3}{2 T_{r}}
$$

In a grid-tied inverter system, both $v_{d c}$ and $E_{d}$ cannot be equal to zero, then the term $G^{-1}(x)$ exists. Similarly to the previous section, in the case of a step response, it can be shown that the nominal DC-link voltage loop is a typical first-order system, which is expressed as follows

$$
\frac{v_{d c}}{v_{d c r e f}}=\frac{K_{0}}{s+K_{0}}
$$

Therefore, the predictive time $T_{r}$ can be specified based on the desired set-point tracking response defined by (31). The disturbance can be estimated in the same way as for the innerloop shown in Sect. IV. That is,

$$
\left\{\begin{array}{l}
\hat{b}_{v}(t)=-\mu_{v} K_{0} \int_{0}^{t} e_{v}(\tau) d \tau-\mu_{v} e_{v}(t)+\mu_{v} e_{v}(0) \\
e_{v}(t)=v_{d c r e f}(t)-v_{d c}(t)
\end{array}\right.
$$

where the observer error dynamics is given by

$$
\dot{e}_{b v}(t)=\frac{\mu_{v}}{C} e_{b v}(t) ; \quad e_{b v}(t)=b_{v}(t)-\hat{b}_{v}(t)
$$

Hence, the disturbance observer (32) can be made exponentially stable if the constant observer gain $\mu_{v}$ is chosen to be negative. More specifically, the disturbance observer works as a first-order low-pass filter; with a time constant equal to $-C / \mu_{v}$. Now, substituting (32) into (29) gives the nonlinear PI predictive controller as follows

$i_{d r e f}(t)=P_{v}\left(v_{d c}\right) e_{v}(t)+I_{v}\left(v_{d c}\right) \int_{0}^{t} e_{v}(\tau) d \tau+N_{v}\left(v_{d c}\right)$

where

$$
P_{v}\left(v_{d c}\right)=-\frac{2 v_{d c}}{3 E_{d}}\left(C K_{0}-\mu_{v}\right) ; \quad I_{v}\left(v_{d c}\right)=\frac{2 v_{d c}}{3 E_{d}} \mu_{v} K_{0}
$$


and

$$
N_{v}\left(v_{d c}\right)=-\frac{2 C v_{d c}}{3 E_{d}} \dot{v}_{d c r e f}-\frac{2 v_{d c}}{3 E_{d}} \mu_{v} e_{v}(0)
$$

The nonlinear predictive term $N_{v}\left(v_{d c}\right)$ has the advantage to achieve a high performance trajectory tracking if the control objective is to follow a predefined time-varying reference [24]. For PV applications, the goal is to regulate the DC-link voltage at a desired steady-state level rather than tracking a fast time-varying reference. For a constant set-point, i.e., $\dot{v}_{d c r e f}=0$, the term $N_{v}\left(v_{d c}\right)$ allows recovering approximately the nominal tracking performance defined by (31) since it considers the information about the initial tracking error. Such an information is not generally included in the conventional PI controller. This explains why the proposed controller is superior to the PI controller.

\section{Closed-loop System ANd the Design PARAMETERS}

Neglecting the initial tracking error $e_{d, q, v}(0)$, the dynamic error of the closed-loop system, for the inner-loop, under the composite controller is governed by

$$
\left\{\begin{array}{l}
\dot{e}_{d}+\left(K_{0}-\frac{\mu_{d}}{L}\right) e_{d}(t)-\frac{K_{0} \mu_{d}}{L} \int_{0}^{t} e_{d}(\tau) d \tau-\frac{1}{L} b_{d}=0 \\
\dot{e}_{q}+\left(K_{0}-\frac{\mu_{q}}{L}\right) e_{q}(t)-\frac{K_{0} \mu_{q}}{L} \int_{0}^{t} e_{q}(\tau) d \tau-\frac{1}{L} b_{q}=0
\end{array}\right.
$$

and that of the outer-loop is given by

$$
\dot{e}_{v}+\left(K_{0}-\frac{\mu_{v}}{C}\right) e_{v}(t)-\frac{K_{0} \mu_{v}}{C} \int_{0}^{t} e_{v}(\tau) d \tau-\frac{1}{C} b_{v}=0
$$

Combining (17) with (37) and (38), leads to the following closed-loop system characteristic equations

$$
\left\{\begin{array}{l}
s^{2}+\left(K_{0}-\frac{\mu_{d, q}}{L}\right) s-\frac{K_{0} \mu_{d, q}}{L}=0 \\
s^{2}+\left(K_{0}-\frac{\mu_{v}}{C}\right) s-\frac{K_{0} \mu_{v}}{C}=0
\end{array}\right.
$$

Therefore, the poles of the outer-loop are $\frac{\mu_{d, q}}{L}$ and $-K_{0}$ while those of the inner-loop are $\frac{\mu_{v}}{C}$ and $-K_{0}$. As the predictive time is positive and the observer gain is negative, it is clear that the closed-loop stability is guaranteed for both loops separately. Elimination of the steady-state error is achieved by integral action. The parameters of the controller can be set according to the desired pole locations. More specifically, the referenceto-output transfer function for the outer-loop is given by

$$
\frac{v_{d c}}{v_{d c r e f}}=\frac{\left(K_{0}-\frac{\mu_{v}}{C}\right) s-K_{0} \frac{\mu_{v}}{C}}{s^{2}+\left(K_{0}-\frac{\mu_{v}}{C}\right) s-K_{0} \frac{\mu_{v}}{C}}
$$

and the reference-to-output transfer function for the inner loop can be expressed as

$$
\frac{i_{d}}{i_{\text {dref }}}=\frac{i_{q}}{i_{\text {qref }}}=\frac{\left(K_{0}-\frac{\mu_{d, q}}{L}\right) s-K_{0} \frac{\mu_{d, q}}{L}}{s^{2}+\left(K_{0}-\frac{\mu_{d, q}}{L}\right) s-K_{0} \frac{\mu_{d, q}}{L}}
$$

To guarantee the stability of the cascaded structure, the innerloop should be designed so as to have faster response compared to that of the outer-loop. This can be accomplished by selecting the parameters of the controller so that the inner-loop settling time $t_{s i}$, resulted from (41), is much lower than the outer-loop settling time $t_{s o}$, resulted from (40). On the other side, as pointed out in [30], the bandwidth of the inner-loop is limited by the maximum switching frequency of the semiconductor devices, indicating that the settling time $t_{s i}$ cannot be less than the switching period $T_{s w}=1 / f_{s w}$, where $f_{s w}$ is the switching frequency. A minimum value of $t_{s i}=5 T_{s w}$ is usually considered when selecting the bandwidth of the current control [30]. As the design method is based on Taylor series expansion, one can select the predictive time to be sufficiently short based on the desired nominal tracking performance, which may vary according to the system under investigation, the sampling frequency and the performances specification. In addition, the observer gain can be chosen as high as possible to a have a fast disturbance estimation.

Remark 1: A large observer gain permits to obtain a fast disturbance rejection but at the same time amplifies the measurement noises, causing a severe degradation of the grid power quality by increasing the total harmonic distortion (THD). Accordingly, the grid power quality requirement limits the performance of the closed-loop system. This means that, for renewable energy applications, the observer gain should be chosen to overcome the tradeoff between a fast disturbance rejection response and a low total harmonic distortion.

Remark2: A short predictive time results in excellent setpoint tracking performance but leads to a large control effort. To overcome such a drawback, the desired set-point tracking response defined by (31) must be chosen adequately so that the line filter current does not exceed its limit value during the transients.

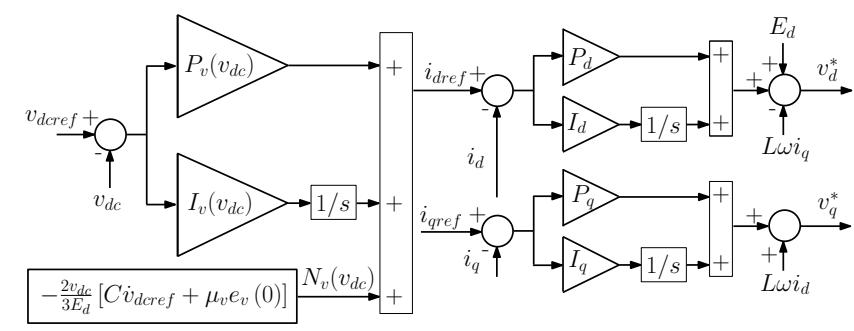

Fig. 3. Block diagram of the proposed NPIPC

\section{SimUlation AND EXPERIMENTAL RESULTS}

The proposed approach is applied for photovoltaic system, which is connected to the grid-tie inverter via a dc-dc converter as shown in Fig. 1. In this configuration, the PV output power is controlled by regulating the PV output voltage through the dc-dc converter. The specific block diagram for the implementation of the proposed control scheme is inspired from Fig. 2, where the third harmonic injection PWM approach is adopted to realize the three-phase voltage commands $v_{a}^{*}, v_{b}^{*}$ and $v_{c}^{*}$ generated by the controller approach. As pointed out above, the inner current control loop can be reduced to decoupled PI controllers and the outer voltage control loop is equivalent to a nonlinear PI controller plus an additional term that depends on the initial tracking error $e_{v}(0)$, resulting in the simplified block diagram depicted in Fig. 3. The proposed controller is experimentally implemented by using the dSPACE DS1103 DSP board with Power PC 750GX Master processor running at $1 \mathrm{GHz}$. The test bed setup is depicted in Fig. 4; it consists of a $2 \mathrm{~kW}$ PV emulator, DC-link capacitor, three-phase IGBT 
with drivers, line filter, and step up transformer which connects the system to the power grid. The system parameters are listed in the Appendix. The sampling period is equal to $1 \mathrm{~ms}$. The period of the PWM signal is chosen equal to $T_{s w}=0.2 \mathrm{~ms}$. Following remarks 1 and 2, and the abovementioned considerations related to the design parameters and presented in Section IV, the predictive time $T_{r}$ for the inner and the outer loops are set to be equal to $0.8 \mathrm{~ms}$ and $10 \mathrm{~ms}$, respectively. The observer gains $\mu_{d}, \mu_{q}$ and $\mu_{v}$ are set to be equal -0.2 . As a result, $t_{s i}=9 T_{s w}$ and $t_{s o} \approx 18 t_{s i}$, meaning that the minimum value of $t_{s i}$ is respected, and the closedloop response of the inner-loop is much faster than that of the outer-loop.

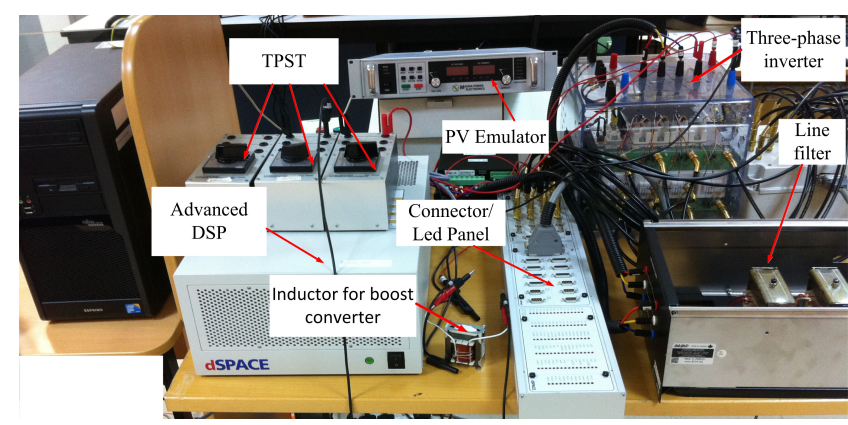

Fig. 4. Experimental setup for performance testing of the proposed controller.

\section{A. Simulation Results}

Here, Matlab/Simulink software package is used to carry out the computer simulation. The objective of this test is to compare the performance of the proposed controller with a classical PI controller at the startup phase. As seen in Fig. 3, the proposed control scheme reduces to a nonlinear PI controller for the outer-loop and it is equivalent to a decoupled PI controllers for the inner-loop. Hence, the objective of comparing the proposed topology with a PI controller can be achieved through testing the performance of the control scheme, shown in Fig. 3, with and without the predictive part $N_{v}$, while using the same current control. Such a test can be considered as a fair comparison since both controllers have the same characteristic equation for the closed-loop system. The control objective is to regulate the DC-link voltage at $85 \mathrm{~V}$ while maintaining the $q$-axis current equal to zero in order to achieve unity power factor operation. At the startup phase, the PV system is disconnected from the grid and the inverter operates as a rectifier while the grid voltage stabilizes the DClink voltage at a certain level. At $t=0.04 \mathrm{~s}$, the inverter is enabled to regulate the DC-link voltage at the desired set-point. From Fig. 5, it can be observed that the DC-link voltage response, under the composite controller, successfully follows the desired nominal response (DNR), described by the transfer function (31), while a large overshoot is observed with the conventional PI controller. This confirms that the nonlinear PI predictive controller can guarantee zero steady-state error while, at the same time, preserves the nominal tracking performance. It can also be seen that the $q$-axis current is well controlled under the proposed approach. Note that, it is possible to improve the transient performance of the PI controller by appropriately tuning the controller parameters. However, this may deteriorate the disturbance rejection performance and leads to unfair comparison. More specifically, in a conventional PI controller, a trade-off should be made between set-point tracking and disturbance rejection performances when selecting the parameters of the outer loop; whilst the proposed design process allows to separately specify the nominal tracking and the disturbance rejection performances. This salient feature cannot be achieved with the classical process design [17], [30].

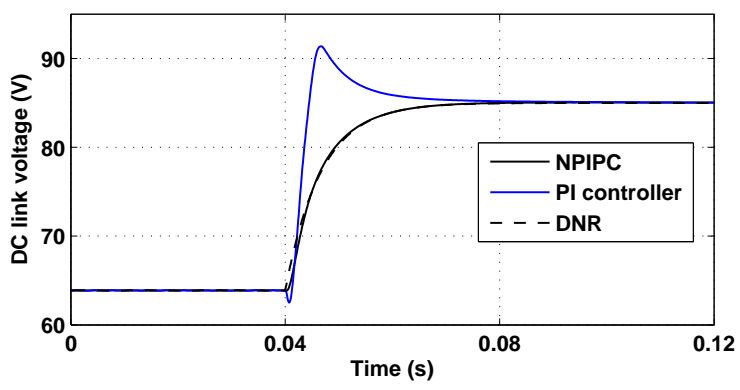

(a) DC link voltage response

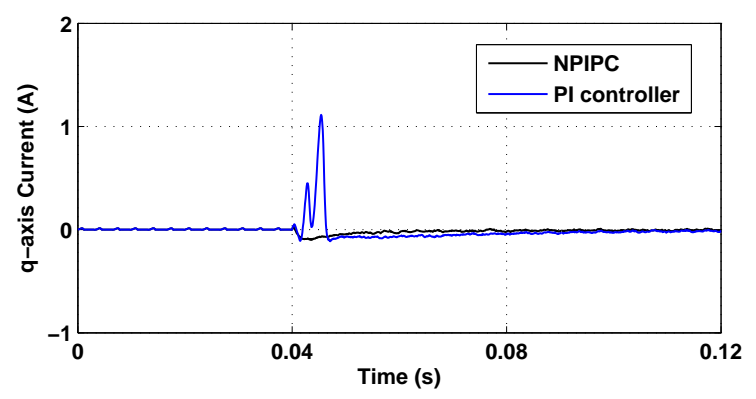

(b) $q$-axis current

Fig. 5. Simulation results: Step response during the startup phase under both the proposed controller and the convectional PI controller

\section{B. Experimental Results}

For the experiments, the Tektronix high voltage differential probe (P5 200) and the Tektronix current probe amplifier (TCP A300) were used in conjunction with the Tektronix 4104B $\mathrm{L}$ digital phosphor oscilloscope to display the experimental results while the Fluke 435- II power quality analyzer was utilized to measure the total harmonic current distortion. A proper signal conditioning is used to measure the required voltage and currents for real-time implementation. The parameters of the controller are chosen similar to those used for the simulation.

1) Nominal Tracking Performance: Figure 6, shows the experimental results with the proposed approach and the PI controller. The proposed approach approximately maintains the desired performance, but, with the PI controller, the DClink voltage response includes a significant overshoot, which may cause the line filter current to exceed its limit during transients. Under the proposed controller, the line filter current is well controlled during the transients because the desired closed-loop response is chosen adequately. Hence, no saturation blocks are required to limit the current, which necessitate 
the design of an anti-windup scheme. By considering the unavoidable mismatch between the simulator and the laboratory setup, overall, it can be concluded that the experimental results are consistent with the simulation results and the theoretical analysis.

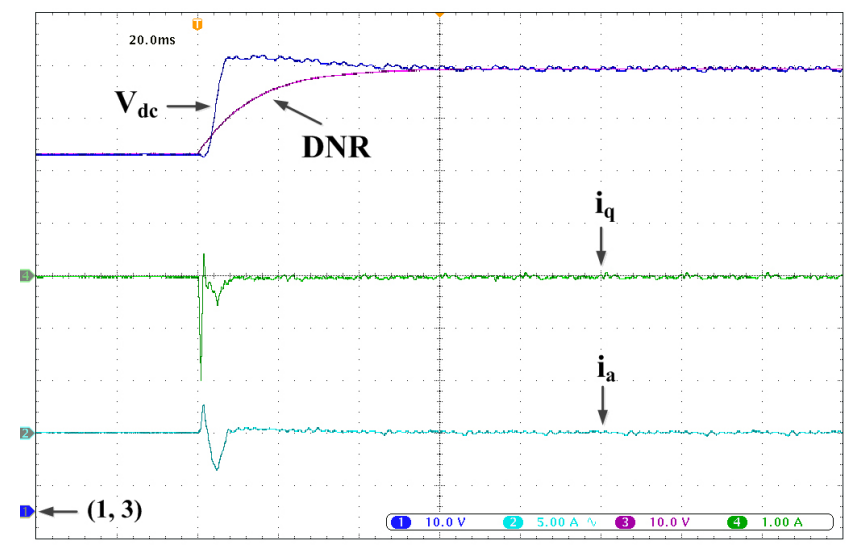

(a) Experimental results under PI controller

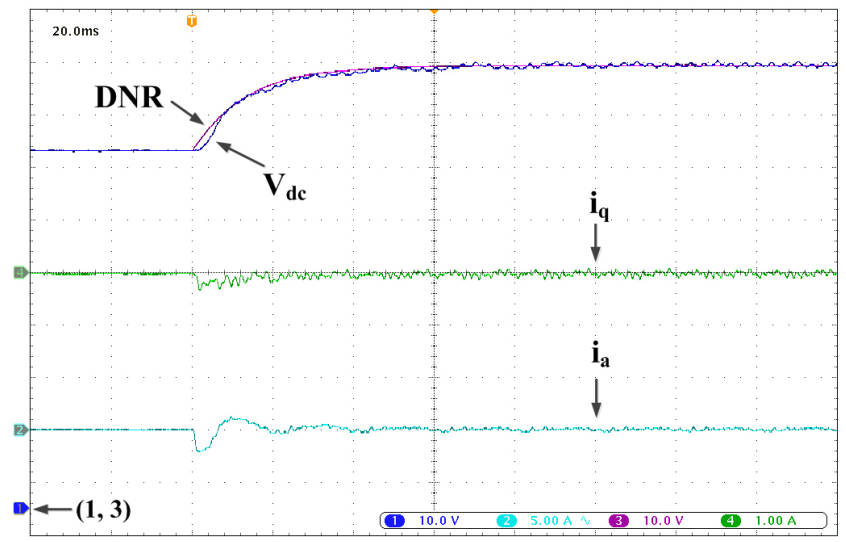

(b) Experimental results under the proposed controller, i.e., NPIPC

Fig. 6. Experimental results: Step response during the startup phase under both the proposed controller and the convectional PI controller

2) Disturbance Rejection Performance Under Abrupt Disturbance: It is clear that the proposed controller has the ability to cope with model uncertainty and unknown disturbance since it contains an integral action. But, this test was performed to analyse the effect of the observer gain on the disturbance rejection performance, and to investigate the dynamic performance in response to a sudden change in the active power. The major disturbance that can affect the system is the variation of the delivered active power because of the changes in atmospheric conditions, which can have an impact on the DC-link voltage regulation. That is why, a disturbance rejection performance of the proposed approach was tested under a step change in delivered active power using two values of $\mu_{v} ; \mu_{v}=-0.05$ and $\mu_{v}=-0.2$. In this case, the DC-link voltage reference was kept constant at $85 \mathrm{~V}$ while the PV power output was suddenly stepped from $650 \mathrm{~W}$ to zero at $t=0.5 \mathrm{~s}$, to test the performance of the proposed controller under a severer condition. This is done by an abrupt disconnection of the PV system from the grid by using a Triple-Pole-Single-Throw (TPST) knife.

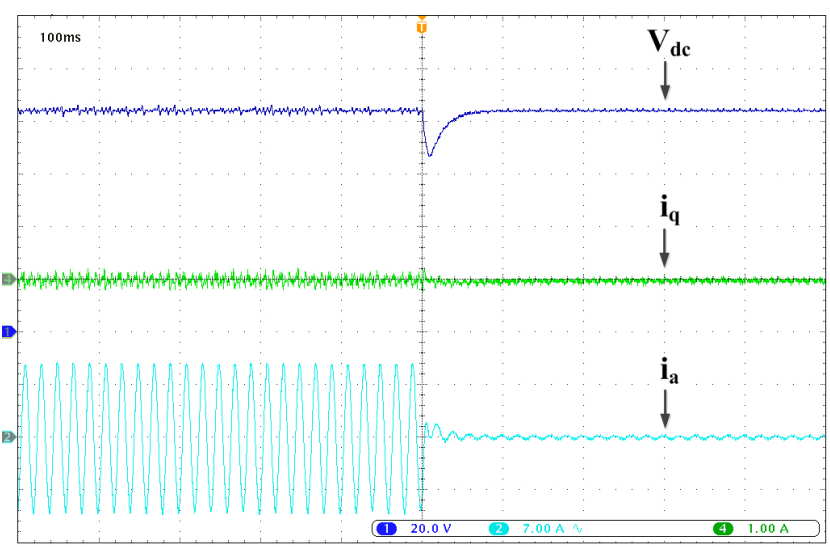

(a) Disturbance rejection performance with $\mu_{v}=-0.2$

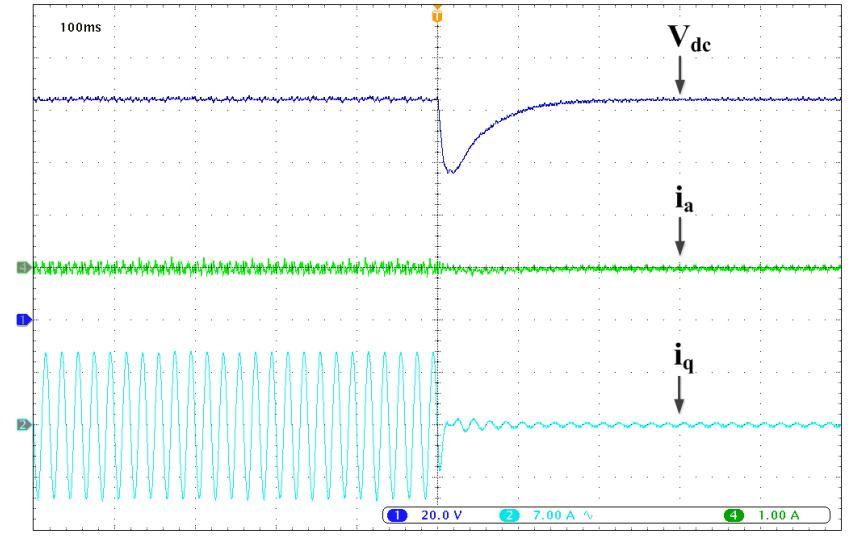

(b) Disturbance rejection performance with $\mu_{v}=-0.05$

Fig. 7. Experimental results: system's response under a sudden disconnection of the PV system from the grid.

From Fig. 7, it can be seen that, at $t=0.5 \mathrm{~s}$, the DC-link voltage decreased, and it took a few milliseconds to return to its steady-state value. However, it can be observed that the disturbance rejection performance is improved by increasing the observer gain. This is because a large observer gain causes the disturbance estimation to reach quickly its steady-state value, and modifies the control to eliminate the offset caused by the disturbance. It can also be observed in these plots that the $q$-axis current is well regulated to its reference value and it is robust against the sudden change in the active power. More interestingly, the disturbance observer $b_{v}$, which estimates the PV current, converges to a stable steady-state condition within a time constant that is specified by the observer gain, which confirms the theoretical analysis. However, a large observer gain relatively magnifies the measurement noises as shown in Fig. 8(a) with comparison to Fig. 8(b). Therefore, it is clear that a larger observer gain degrades the level of grid power quality by increasing the THD of the grid current as shown in the THD graphs (Figs. 9 and 10) and summarized in the Table I, and hence attention should be given for practical implementation of the proposed controller. 


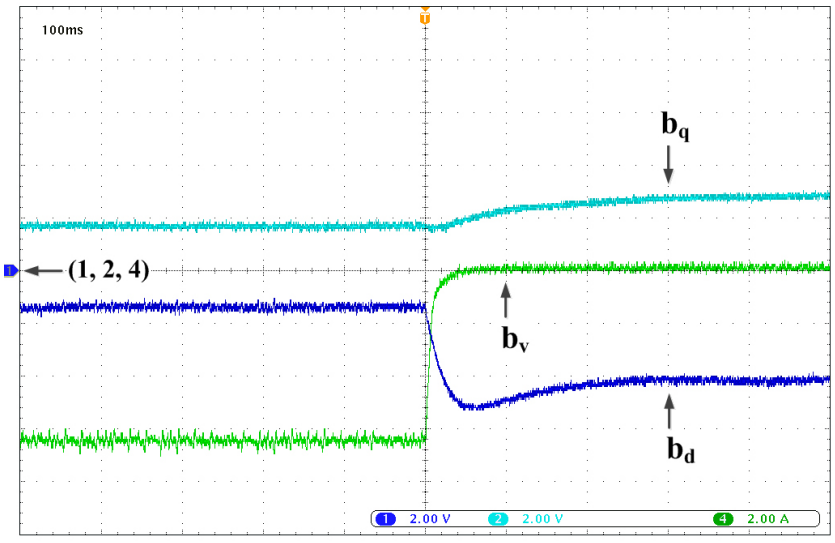

(a) Nonlinear disturbance observer response with $\mu_{v}=-0.2$

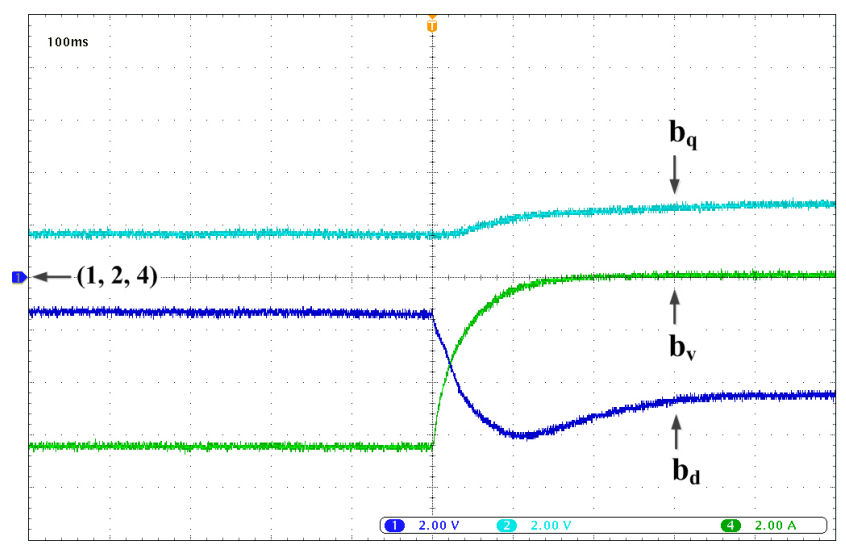

(b) Nonlinear disturbance observer response with $\mu_{v}=-0.05$

Fig. 8. Experimental results: Nonlinear disturbance observer response under a sudden disconnection of the PV system from the grid.

3) Performance Evaluation Under Change of Reactive Power Set-point: Test under changes in reactive power have been also conducted with the active power maintained constant at about $350 \mathrm{~W}$, and Fig. 11 shows the corresponding results. Figs. 12 and 13 give the THD graphs of the current before and after application of step change in reactive power, respectively. As seen, the $q$-axis current was stepped down from 0 A to $2.5 \mathrm{~A}$ at $t=0.05 \mathrm{~s}$. The $q$-axis current is selected to be negative in order to inject the reactive power in the grid. This operation is required in responding to the request from grid operator to maintain grid voltage at desired level. From the results, it can be observed that the DC-link voltage is almost insensitive to changes in reactive power. The $q$-axis current reaches rapidly its new steady-state value in response to a step change within the specified settling time, which is about $1.8 \mathrm{~ms}$. The current waveform is maintained in phase with the phase voltage $e_{a}$ when $q$-axis current is equal to zero. This means that unity power factor operation is ensured during the period when the $q$-axis current reference is equal to zero. However, it is clear that a change in $q$-axis current reference affects the phase-shift between the phase current and voltage as the delivered reactive power becomes different from zero. From Figs. 12 and 13 , it can be observed that the THD of the current is below 5\%, which is in accordance with the grid connection requirements [31]. As mentioned above, the current control loop is equivalent to the conventional decoupled PI controllers that are usually adopted for the grid-tied inverters [17], but the design process is different. Moreover, it is noticed that, at steady-state regime, the outer voltage loop reduces to a conventional PI controller. This explains why the proposed controller is not compared with the classical PI controller in terms of the steady-state performance such as the THD of the current.

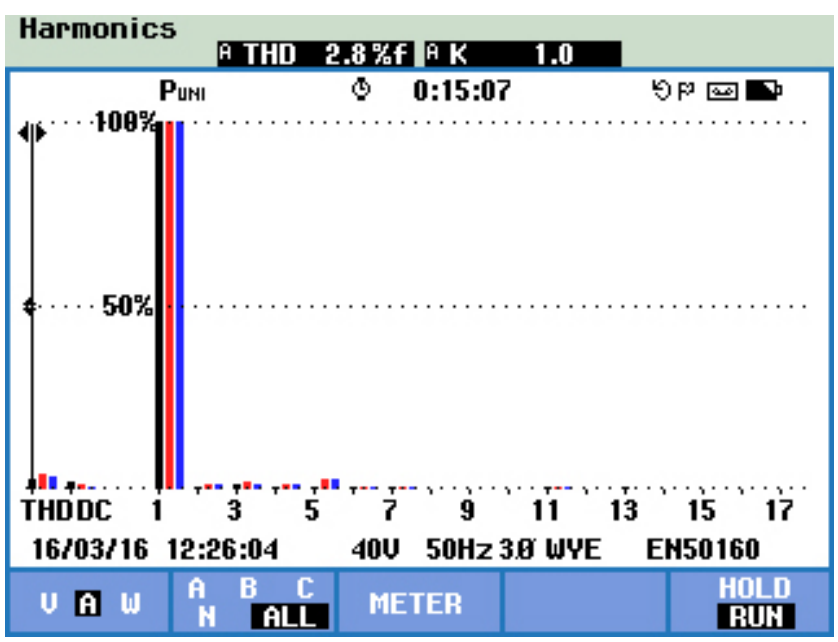

Fig. 9. Current THD under maximal active power with $\mu_{v}=-0.2$.

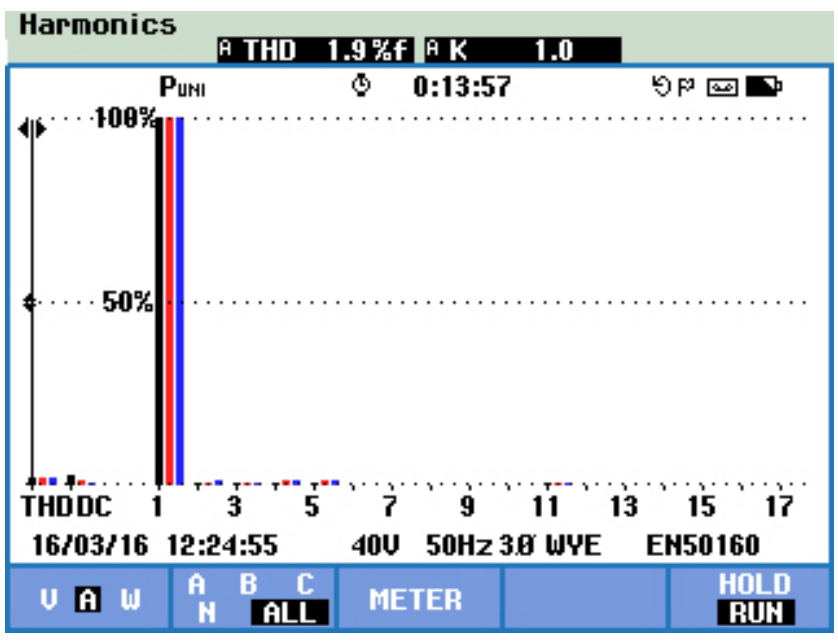

Fig. 10. Current THD under maximal active power with $\mu_{v}=-0.05$.

TABLE I

THD OF THE LINE CURRENT

\begin{tabular}{|c||c||c|}
\hline$\mu_{v}$ & -0.05 & -0.2 \\
\hline THD \% & 1.9 & 2.8 \\
\hline
\end{tabular}

Performance testing under changes in reactive power was realized, especially, to partially demonstrate the ability of the proposed controller to deal with low voltage right thorough (LVRT) capability, which requires reactive power support. In such a situation, the control scheme, presented in [1] and consisting of the inner loop and outer loop controllers, may be combined with the proposed design process to improve the performance of the grid-tied PV system during grid voltage dips. 


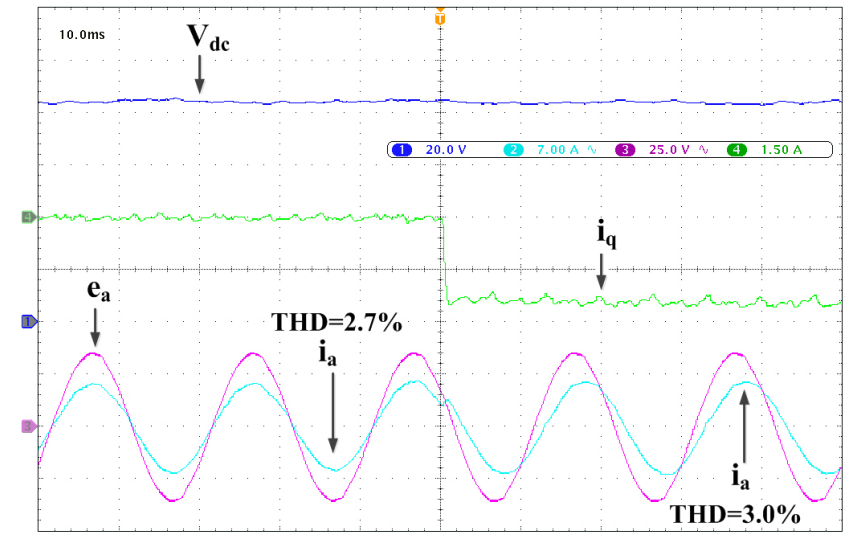

Fig. 11. Experimental results: System's response to changes in reactive power.

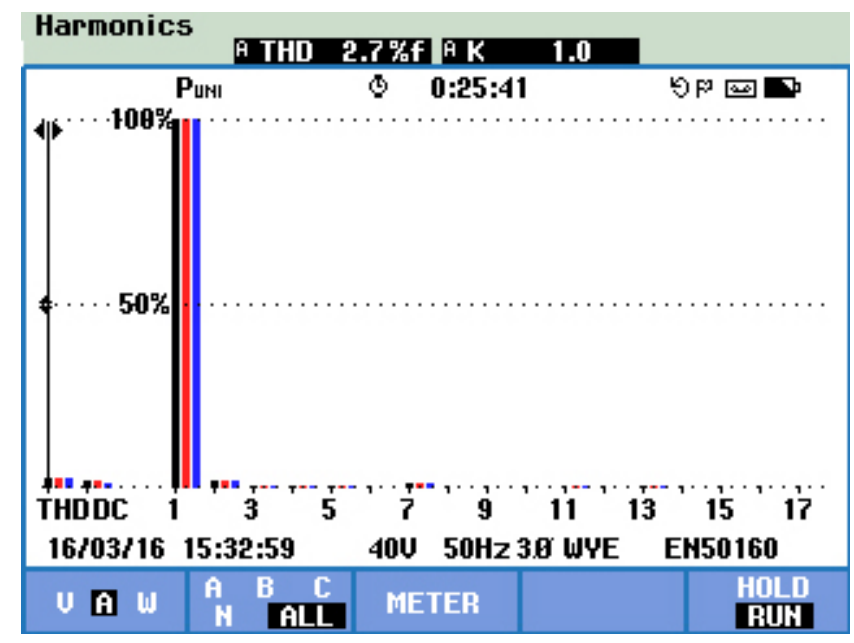

Fig. 12. Current THD under active power exchange of $350 \mathrm{~W}$.

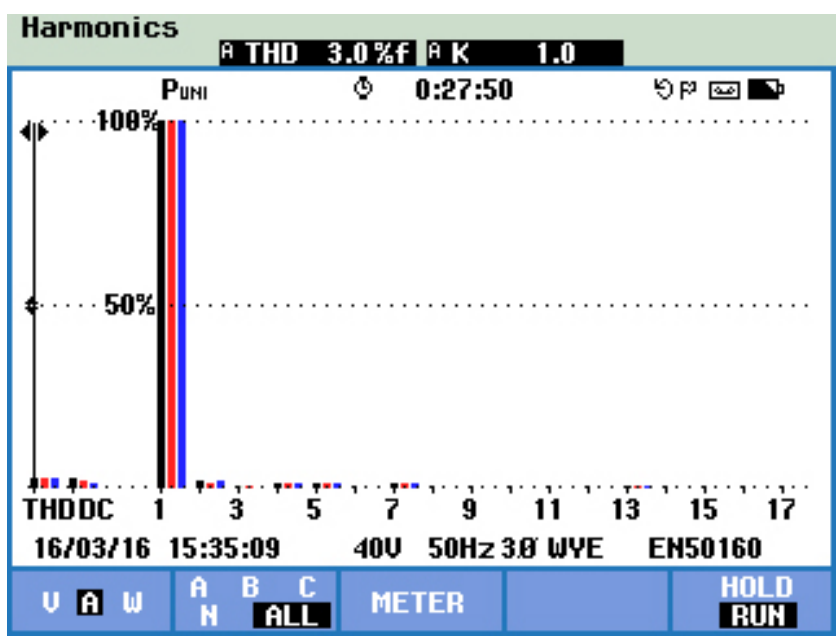

Fig. 13. Current THD under active and reactive powers exchange.

\section{CONCLUSION}

A nonlinear PI predictive controller has been proposed to control a three-phase grid-tied inverter used in photovoltaic system. The paper exposed analytically how to design the nonlinear PI predictive controller by means of combining a continuous-time model predictive control with a nonlinear disturbance observer. The design method enabled us to specify the set-point tracking and the disturbance attenuation performances independently. Another promising feature lies in the use of a disturbance observer, which can be utilized for other objectives such as islanding or fault detection.

Simulation and experimental results have been provided showing the effectiveness of the proposed strategy. It is also found that the proposed approach can provide better performance in comparison to a conventional PI controller during the startup phase. The proposed approach is convenient for practical implementation and it provides practitioners with an alternative method to design PI controllers for three-phase grid-connected renewable energy resources using an $L$ filter, with a possible extension to applications using an $L C L$ filter.

\section{APPENDIX}

The parameters of the grid-tie inverter and the $\mathrm{PV}$ emulator are $V_{d c}=85 \mathrm{~V}, E_{d}=33 \mathrm{~V}, L=6.8 \mathrm{mH}, C=1.052 \mathrm{mF}$ and $\omega=314.5 \mathrm{rad} / \mathrm{s}$. For PV emulator, the maximum power output, the short circuit current and the open circuit voltage are $650 \mathrm{~W}, 11 \mathrm{~A}$ and $80 \mathrm{~V}$, respectively. The PV voltage at the maximum power is about $65 \mathrm{~V}$.

It is noticed that because of our laboratory test setup, it was not possible to use the actual grid voltage of $415 \mathrm{~V}$. More specifically, the grid side voltage is dictated by many factors such as the DC-link voltage, the characteristic of the converter itself (two-level), the line filter, the power exchange, and the modulation technique employed. More information about the relationship between the grid side converter's limit and the grid voltage can be found in Section 2.4 in [32].

\section{REFERENCES}

[1] F. Yang, L. Yang, and X. Ma, "An advanced control strategy of PV system for low-voltage ride-through capability enhancement," Solar Energy, vol. 109, pp. 24-35, Sep 2014.

[2] K.-H. Kim, Y.-C. Jeung, D.-C. Lee, and H.-G. Kim, "LVRT scheme of PMSG wind power systems based on feedback linearization," IEEE Trans. Power Electron., vol. 27, no. 5, pp. 2376-2384, May 2012.

[3] D.-E. Kim and D.-C. Lee, "Feedback linearization control of three-phase UPS inverter systems," IEEE Trans. Ind. Electron., vol. 57, no. 3, pp. 963-968, March 2010.

[4] M. Mahmud, H. Pota, and M. Hossain, "Dynamic stability of threephase grid-connected photovoltaic system using zero dynamic design approach," IEEE J. Photovolt., vol. 2, no. 4, pp. 564-571, Oct 2012.

[5] M. Mahmud, H. Pota, M. Hossain, and N. Roy, "Robust partial feedback linearizing stabilization scheme for three-phase grid-connected photovoltaic systems," IEEE J. Photovolt., vol. 4, no. 1, pp. 423-431, Jan 2014.

[6] F. Delfino, F. Pampararo, R. Procopio, and M. Rossi, "A feedback linearization control scheme for the integration of wind energy conversion systems into distribution grids," IEEE J. Sys., vol. 6, no. 1, pp. 85-93, March 2012.

[7] C. Xia, Q. Geng, X. Gu, T. Shi, and Z. Song, "Input output feedback linearization and speed control of a surface permanent-magnet synchronous wind generator with the boost-chopper converter," IEEE Trans. Ind. Electron., vol. 59, no. 9, pp. 3489-3500, Sep 2012.

[8] X. Bao, F. Zhuo, Y. Tian, and P. Tan, "Simplified feedback linearization control of three-phase photovoltaic inverter with an LCL filter," IEEE Trans. Power Electron., vol. 28, no. 6, pp. 2739-2752, June 2013.

[9] J. Hu, J. Zhu, and D. G. Dorrell, "Model Predictive Control of GridConnected Inverters for PV Systems With Flexible Power Regulation and Switching Frequency Reduction," IEEE Trans. Ind Appl., vol. 51, no. 1, pp. 587-594, Jan 2015. 
[10] Z. Song, C. Xia, and T. Liu, "Predictive current control of three-phase grid-connected converters with constant switching frequency for wind energy systems," IEEE Trans. Ind. Electron., vol. 60, no. 6, pp. 24512464, June 2013.

[11] M. Shadmand, R. Balog, and H. Abu-Rub, "Model predictive control of PV sources in a smart DC distribution system: Maximum power point tracking and droop control," IEEE Trans. Energy Conv., vol. 29, no. 4, pp. 913-921, Dec 2014.

[12] V. Yaramasu, B. Wu, S. Alepuz, and S. Kouro, "Predictive control for low-voltage ride-through enhancement of three-level-boost and NPCconverter-based PMSG wind turbine," IEEE Trans. Ind. Electron., vol. 61, no. 12, pp. 6832-6843, Dec 2014.

[13] J. Espi Huerta, J. Castello-Moreno, J. Fischer, and R. Garcia-Gil, "A synchronous reference frame robust predictive current control for threephase grid-connected inverters," IEEE Trans. Ind. Electron., vol. 57, no. 3, pp. 954-962, March 2010.

[14] V. Yaramasu and B. Wu, "Model predictive decoupled active and reactive power control for high-power grid-connected four-level diode-clamped inverters," IEEE Trans. Ind. Electron., vol. 61, no. 7, pp. 3407-3416, July 2014

[15] P. E. Kakosimos, A. G. Kladas, and S. N. Manias, "Fast photovoltaicsystem voltage-or current-oriented MPPT employing a predictive digital current-controlled converter," IEEE Trans. Ind. Electron., vol. 60, no. 12 pp. 5673-5685, Dec 2013.

[16] H. M. Hasanien and S. Muyeen, "A taguchi approach for optimum design of proportional-integral controllers in cascaded control scheme," IEEE Trans. Power Electron., vol. 28, no. 2, pp. 1636-1644, May 2013.

[17] R. Kadri, J.-P. Gaubert, and G. Champenois, "An improved maximum power point tracking for photovoltaic grid-connected inverter based on voltage-oriented control," IEEE Trans. Ind. Electron., vol. 58, no. 1, pp. 66-75, Jan 2011.

[18] V. Blasko and V. Kaura, "A new mathematical model and control of a three-phase AC-DC voltage source converter," IEEE Trans. Power Electron., vol. 12, no. 1, pp. 116-123, Jan 1997.

[19] J. Dannehl, C. Wessels, and F. W. Fuchs, "Limitations of voltageoriented PI current control of grid-connected PWM rectifiers with filters," IEEE Trans. Ind. Electron., vol. 56, no. 2, pp. 380-388, Feb 2009.

[20] M. Castilla, J. Miret, A. Camacho, J. Matas, D. Vicuna, and L. García, "Reduction of current harmonic distortion in three-phase grid-connected photovoltaic inverters via resonant current control," IEEE Trans. Ind. Electron., vol. 60, no. 4, pp. 1464-1472, April 2013.

[21] A. Kuperman, "Proportional-Resonant Current Controllers Design Based on Desired Transient Performance," IEEE Trans. Power Electron. vol. 30, no. 10, pp. 5341-5345, Oct 2015.

[22] W.-H. Chen, D. J. Ballance, and P. J. Gawthrop, "Optimal control of nonlinear systems: a predictive control approach," Automatica, vol. 39, no. 4, pp. 633-641, April 2003.

[23] W.-H. Chen, D. J. Ballance, P. J. Gawthrop, J. J. Gribble, and J. O'Reilly, "Nonlinear PID predictive controller," IEE Proc. Control Theory Appl., vol. 146, no. 6, pp. 603-611, Nov 1999

[24] R. Errouissi, M. Ouhrouche, W.-H. Chen, and A. M. Trzynadlowski, "Robust cascaded nonlinear predictive control of a permanent magnet synchronous motor with antiwindup compensator," IEEE Trans. Ind. Electron., vol. 59, no. 8, pp. 3078-3088, Aug 2012.

[25] C. Wang, X. Li, L. Guo, and Y. W. Li, "A nonlinear-disturbanceobserver-based DC-bus voltage control for a hybrid AC/DC microgrid," IEEE Trans. Power Electron., vol. 29, no. 11, pp. 6162-6177, Nov 2014.

[26] Y. I. Son, I. H. Kim, D. S. Choi, and H. Shim, "Robust cascade control of electric motor drives using dual reduced-order PI observer," IEEE Trans. Ind. Electron., vol. 62, no. 6, pp. 3672-3682, June 2015.

[27] J. Yang, W. Zheng, S. Li, B. Wu, and M. Cheng, "Design of a prediction accuracy enhanced continuous-time MPC for disturbed systems via a disturbance observer," IEEE Trans. Ind. Electron., vol. 62, no. 9, pp. 5807-5816, Sep 2015.

[28] S. Khajehoddin, M. Karimi-Ghartemani, P. Jain, and A. Bakhshai, "A control design approach for three-phase grid-connected renewable energy resources," IEEE Trans. Syst. Energy, vol. 2, no. 4, pp. 423-432, Oct 2011.

[29] V. Kaura and V. Blasko, "Operation of a phase locked loop system under distorted utility conditions," IEEE Trans. Ind. Appl., vol. 33, no. 1, pp. 58-63, Jan 1997.
[30] P. Verdelho and G. Marques, "DC voltage control and stability analysis of PWM-voltage-type reversible rectifiers," IEEE Trans. Ind. Electron., vol. 45, no. 2, pp. 263-273, April 1998.

[31] "Standard for Interconnecting Distributed Resources With Electric Power Systems," IEEE Std 1547-2003, pp. 1-28, July 2003.

[32] G. Abad, J. Lopez, M. Rodríguez, L. Marroyo, and G. Iwanski, Doubly fed induction machine: modeling and control for wind energy generation. New York, NY, USA: John Wiley \& Sons, Sep 2011,

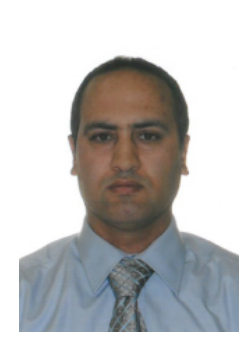

Rachid Errouissi (M'15) received the B.Sc. degree in electronics from the Faculty of Sciences and Technology of Mohammedia, Morocco, in 1998; the M.Sc. degree in power electronics from École Mohammadia d'ingénieurs in Rabat, Morocco, in 2001; the double M.Sc. degrees in electrical engineering and in automation and system engineering from University Claude Bernard, Lyon, France, in 2002 and 2004; and the Ph.D. degree in electrical engineering from the University of Quebec, Chicoutimi, QC, Canada, in 2010. From 2011 to 2014, he worked as a Postdoctoral Researcher with the Department of Electrical and Computer Engineering, University of New Brunswick. Since 2014, he has been with the Petroleum Institute, Abu Dhabi, UAE, where he is currently conducting research works in renewable energy and advanced control systems. His area of interests include advanced control, nonlinear control, electric machines and drives, and renewable energy conversion systems. He is a registered Professional Engineer in the province of New Brunswick, Canada.

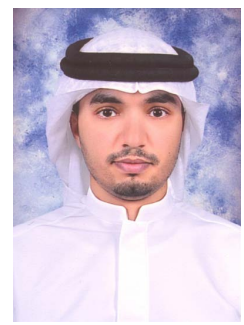

Ahmed Al-Durra (S'07-M'10-SM'14) received the B.S., M.S., and PhD in Electrical and Computer Engineering from the Ohio State University (OSU) in 2005, 2007, and 2010, respectively. He conducted his $\mathrm{PhD}$ research at the Center for Automotive Research in OSU on the applications of modern estimation and control theories to automotive propulsion systems. At the present, he is an Associate Professor in the Electrical Engineering Department at the Petroleum Institute, Abu Dhabi, UAE. He obtained the PI Research \& Scholarship Award for Junior Faculty in 2014 His research interests are application of estimation and control theory in power system stability, Micro and Smart Grids, renewable energy, and process control. He has published over 80 scientific articles in Journals, International Conferences, and book chapters. He has successfully accomplished several research projects at international and national levels. He has supervised/co-supervised over $20 \mathrm{PhD} /$ Master students. $\mathrm{He}$ is the co-founder of Renewable Energy Laboratory at the Petroleum Institute.

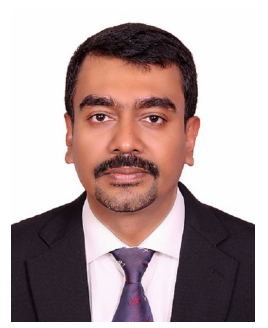

S. M. Muyeen (S'03-M'08-SM'12) received his B.Sc. Eng. Degree from Rajshahi University of Engineering and Technology (RUET), Bangladesh formerly known as Rajshahi Institute of Technology, in 2000 and M. Eng. and Ph.D. Degrees from Kitami Institute of Technology, Japan, in 2005 and 2008, respectively, all in Electrical and Electronic Engineering. At the present, he is working as Associate Professor in Electrical Engineering Department at the Petroleum Institute, Abu Dhabi. His research interests are power system stability and control, electrical machine, FACTS, energy storage system (ESS), Renewable Energy, and HVDC system. He has been a Keynote Speaker and an Invited Speaker at many international conferences, workshops, and universities. He has published over 150 articles in different journals and international conferences. He has published five books as an author or editor. Dr. Muyeen is the senior member of IEEE 